Draft VERSiOn July 11, 2012

Preprint typeset using $\mathrm{IAT}_{\mathrm{E}} \mathrm{X}$ style emulateapj v. 5/2/11

\title{
CORE-COLLAPSE SUPERNOVA EQUATIONS OF STATE BASED ON NEUTRON STAR OBSERVATIONS
}

\author{
A. W. SteineR \\ Institute for Nuclear Theory, University of Washington, Seattle, WA 98195 \\ M. HEMPEL \\ Department of Physics, University of Basel, Klingelbergstrasse 82, 4056 Basel, Switzerland \\ T. FISCHER \\ GSI, Helmholtzzentrum für Schwerionenforschung GmbH, Planckstraße 1, 64291 Darmstadt, Germany \\ Technische Universität Darmstadt, Schlossgartenstrasse 2, 64289 Darmstadt, Germany \\ Draft version July 11, 2012
}

\begin{abstract}
Many of the currently available equations of state for core-collapse supernova simulations give large neutron star radii and do not provide large enough neutron star masses, both of which are inconsistent with some recent neutron star observations. In addition, one of the critical uncertainties in the nucleon-nucleon interaction, the nuclear symmetry energy, is not fully explored by the currently available equations of state. In this article, we construct two new equations of state which match recent neutron star observations and provide more flexibility in studying the dependence on nuclear matter properties. The equations of state are also provided in tabular form, covering a wide range in density, temperature and asymmetry, suitable for astrophysical simulations. These new equations of state are implemented into our spherically symmetric core-collapse supernova model, which is based on general relativistic radiation hydrodynamics with three-flavor Boltzmann neutrino transport. The results are compared with commonly used equations of state in supernova simulations of 15 and $40 \mathrm{M}_{\odot}$ progenitors. We do not find any simple correlations between individual nuclear matter properties at saturation and the outcome of these simulations. However, the new equations of state lead to the most compact neutron stars among the relativistic mean-field models which we considered. The new models also obey the previously observed correlation between the time to black hole formation and the maximum mass of an $s=4$ neutron star.
\end{abstract}

\section{INTRODUCTION}

Core-collapse supernovae (SNe) are some of the most energetic events in the Universe. They release several times $10^{53}$ erg in neutrinos, the gravitational binding energy difference between iron core and neutron star, and in case of SN explosions several $10^{51}$ erg kinetic energy of the ejected material. The latter are related to the standing accretion shock revival, which forms when the super-sonically collapsing stellar core reaches nuclear matter densities and bounces back. The shock wave initially propagates out of the stellar core and thereby loses energy due to the dissociation of infalling heavy nuclei and electron neutrino escapes when it crosses the neutrinospheres. Several explosion mechanisms have been discussed, i.e magneto-rotational by LeBlanc \& Wilson (1970), dissipation of sound waves by Burrows et al. (2006), and the standard scenario driven by neutrino heating by Bethe \& Wilson (1985).

The equation of state (EOS) is one of the critical and highly uncertain microphysical inputs for modeling corecollapse supernovae. The EOS in SN simulations has to handle several intrinsically different regimes. For temperatures below about $0.5 \mathrm{MeV}$, time-dependent nuclear reactions are important in determining the nuclear composition. Above $0.5 \mathrm{MeV}$, nuclear statistical equilibrium can be applied, where the dependence of the EOS can be reduced to the three independent variables: temperature $T$, baryon number density $n_{B}$ and proton-to-baryon ra- tio (or equivalently the electron fraction) $Y_{e}$. Finally, at densities close to and above nuclear matter density, the transition to a state of matter composed of deconfined quarks may take place.

While accurate neutron star mass measurements of double pulsar systems are plentiful, reliable radius measurements have only recently become available. Steiner et al. (2010) obtained quantitative constraints on the EOS of nuclear matter from mass and radius measurements from quiescent low-mass X-ray binaries and objects with photospheric radius expansion bursts. Current neutron star radius observations suggest small radii and lower pressures just above the nuclear saturation density. Small neutron star radii could be interesting for the supernova dynamics, because expects more gravitational binding energy. Unfortunately very few available supernova EOS give (i) nuclear matter properties consistent with those inferred from experiment, (ii) maximum neutron star masses large enough to be consistent with the recent measurement of a 1.97 solar mass neutron star in Demorest et al. (2010), and (iii) masses and radii consistent with recent neutron star observations of e.g. Steiner et al. (2010). Furthermore, most available EOS tables are based on interactions with larger values of the density derivative of the nuclear symmetry energy, $L$, even though lower values are suggested by both intermediate-energy heavy-ion collisions (Tsang et al. 2009), chiral effective field the- 
ory (Hebeler et al. 2010; Tews et al. 2012) and neutron star radii (Steiner \& Gandolfi| 2012; Steiner et al. 2012).

We should note that there are potential systematic uncertainties in the neutron star mass and radius measurements which are not yet taken into account. The relationship between the Eddington flux and the point at which the photosphere returns to the neutron star surface is not under control (Steiner et al. 2010), the evolution of the spectrum during the tail of the burst is not well understood (Suleimanov et al. 2011), and the value of the factor which corrects for the fact that the X-ray spectrum is not a black body may also modify inferred radii. Also, the X-ray spectrum may be modified by accretion and violations of the assumed spherical symmetry. Recent work (Steiner et al. 2012) finds that neutron star radii may be as large as $13 \mathrm{~km}$.

In this article, we construct new EOSs which are both consistent with experimental nuclear data and recent neutron star mass observations. We parameterize nucleonic matter with a new relativistic mean field (RMF) model and describe nuclei and nonuniform nuclear matter with the statistical model from Hempel \& Schaffner-Bielich (2010). The final EOS is also provided in tabular form, covering a wide range in density, temperature, and electron fractions. We apply these EOS tables in core-collapse SN simulations of intermediate- and high-mass progenitors.

\section{EQUATIONS OF STATE}

The most commonly used EOSs are that of Lattimer \& Swesty (1991) (hereafter LS), which is based on the compressible liquid-drop model including surface effects, and H. Shen et al. (1998) (hereafter STOS), which is based on the TM1 RMF interaction (Sugahara \& Toki 1994) and uses the ThomasFermi approximation to describe non-uniform nuclear matter. Both EOSs simplify the baryon composition using the single-nucleus approximation (SNA) for heavy nuclei, and ignore all light nuclei except for alpha particles. There are several studies of the differences between these two EOSs in core-collapse simulations (see, e.g., Sumivoshi et al. 2006, 2007; Fischer et al. 2009; O'Connor \& Ott 2011; Hempel et al. 2012). More recently, several new EOS based on RMF interactions have become available. The new hadronic EOS tables of G. Shen (Shen et al. 2011b,a) are based on NL3 (Lalazissis et al. 1997) and FSUgold (Todd-Rutel \& Piekarewicz 2005) RMF interactions with nuclei described in the Hartree appoximation. The EOS model of Hempel \& Schaffner-Bielich (2010) and Hempel et al. (2012) (hereafter HS) is based on the statistical approach and is also used in the present study. It goes beyond SNA by including the detailed nuclear composition, based on experimentally measured nuclear masses as well as different theoretical mass models. Tables are available for seven different RMF parameterizations, including TM1, FSUGold, and TMA (Toki et al. 1995) and the new parameterizations SFHo and SFHx developed in the present article.

In addition to the hadronic EOS a first-order phase transition to quark matter has been studied in Sagert et al. (2009) and Fischer et al. (2011), where it has been demonstrated that it can trigger explosions even in spherically symmetric supernova models. Note that
TABLE 1

RMF MODEL PARAMETERS

\begin{tabular}{llrlrl}
\hline Quantity & Unit & SFHo & & SFHx & \\
\hline$c_{\sigma}$ & $\mathrm{fm}$ & 3.1780 & & 3.4016 & \\
$c_{\omega}$ & $\mathrm{fm}$ & 2.2726 & & 2.5730 & \\
$c_{\rho}$ & $\mathrm{fm}$ & 2.4047 & & 2.4199 & \\
$\mathrm{~b}$ & & 7.4653 & $\times 10^{-3}$ & 4.8157 & $\times 10^{-3}$ \\
$\mathrm{c}$ & & -4.0887 & $\times 10^{-3}$ & -4.3984 & $\times 10^{-3}$ \\
$\zeta$ & & -1.7013 & $\times 10^{-3}$ & 4.4218 & $\times 10^{-3}$ \\
$\xi$ & & 3.4525 & $\times 10^{-3}$ & 2.0535 & $\times 10^{-4}$ \\
$a_{1}$ & $\mathrm{fm}^{-1}$ & -2.3016 & $\times 10^{-1}$ & -4.6241 & $\times 10^{-1}$ \\
$a_{2}$ & & 5.7972 & $\times 10^{-1}$ & 1.6604 & \\
$a_{3}$ & $\mathrm{fm}$ & 3.4446 & $\times 10^{-1}$ & 1.1792 & $\times 10^{-2}$ \\
$a_{4}$ & $\mathrm{fm}^{2}$ & 3.4593 & & 2.1595 & $\times 10^{1}$ \\
$a_{5}$ & $\mathrm{fm}^{3}$ & 1.3473 & & 1.5478 & \\
$a_{6}$ & $\mathrm{fm}^{4}$ & 6.6061 & $\times 10^{-1}$ & 8.5506 & $\times 10^{-1}$ \\
$b_{1}$ & & 5.8729 & & 8.4606 & \\
$b_{2}$ & $\mathrm{fm}^{2}$ & -1.6442 & & -2.3629 & \\
$b_{3}$ & $\mathrm{fm}^{4}$ & 3.1464 & $\times 10^{2}$ & 4.0622 & $\times 10^{1}$ \\
$m_{\sigma}$ & $\mathrm{fm}^{-1}$ & 2.3714 & & 2.3844 & \\
\hline
\end{tabular}

the hybrid EOSs used result in extremely compact neutron stars. All of these EOS studies in SN simulations explore also very massive progenitor stars which in the end collapse to a black hole, for which the post-bounce time until that moment can be used as an observable to constrain characteristics of the phase transition.

There are several critical parameters for characterizing the equation of state of hadronic matter, and some of the most relevant and yet uncertain parameters are the nuclear incompressibility, $K$, the symmetry energy at the saturation density, $J$, and the logarithmic derivative of the symmetry energy $L$. The compressibility was an important parameter for early corecollapse simulations (Baron et al. 1985), and the compressibilities of the LS EOS tables are available for 180, 200, and $375 \mathrm{MeV}$. However, the lowest and highest of these values are far outside the currently acceptable range of $240 \pm 10 \mathrm{MeV}$ (Colo et al. 2004). We note that there is still some model dependence in extracting this value Piekarewicz (2010); Khan \& Margueron (2012). Early simulations also suggested that the symmetry energy is important (Sumiyoshi et al. 1995)). Recent constraints on $J$ suggest $28<S<34$ from a combination of constraints from experiments, theory, and observations of neutron star masses and radii (Hebeler et al. 2010; Steiner \& Gandolfi 2012; Lattimer \& Lim 2012; Tsang et al. 2012; Steiner et al. 2012; Tews et al. 2012). These works also imply constraints on $L$, but these constraints are more model-dependent and are not always consistent with each other. Only one of the original supernova EOS tables, the LS table with $K=220 \mathrm{MeV}$, obeys the current constraints on these EOS parameters.

For the new EOS SFHo and SFHx, we choose to use a covariant Lagrangian based on the Walecka model where nucleons interact via the exchange of $\sigma, \omega$, and $\rho$ mesons in the mean-field approximation. The non-linear Walecka model is well-known to have a limited range of variation in the isospin sector and the nuclear symmetry energy is controlled entirely through the coupling of the nucleons to the $\rho$ meson. In order to provide more flexibility, several additional terms like $\rho^{4}$ and $\sigma^{2} \rho^{2}$ have been considered. We use the parameterization in Steiner et al. (2005) which provides enough freedom to modify the low- 
TABLE 2

Properties at Saturation density and neutron star properties for the the Different EOSs under investigation. The DEFINITION OF ALL THE QUANTITIES IS GIVEN IN THE TEXT.

\begin{tabular}{|c|c|c|c|c|c|c|c|c|c|c|c|}
\hline EOS & $\begin{array}{c}n_{B}^{0} \\
{\left[\mathrm{fm}^{-3}\right]}\end{array}$ & $\begin{array}{c}E_{0} \\
{[\mathrm{MeV}]}\end{array}$ & $\begin{array}{c}K \\
{[\mathrm{MeV}]}\end{array}$ & $\begin{array}{c}K^{\prime} \\
{[\mathrm{MeV}]}\end{array}$ & $\begin{array}{c}J \\
J \\
{[\mathrm{MeV}]}\end{array}$ & $\begin{array}{c}L \\
{[\mathrm{MeV}]}\end{array}$ & $\begin{array}{c}m_{n}^{*} / m_{n} \\
-\end{array}$ & $\begin{array}{c}m_{p}^{*} / m_{p} \\
-\end{array}$ & $\begin{array}{l}\mathrm{R}_{1.4} \\
{[\mathrm{~km}]}\end{array}$ & $\begin{array}{c}\mathrm{M}_{\mathrm{T}=0, \mathrm{Max}} \\
{\left[\mathrm{M}_{\odot}\right]}\end{array}$ & $\begin{array}{c}\mathrm{M}_{\mathrm{S}=4, \mathrm{Max}} \\
{\left[\mathrm{M}_{\odot}\right]}\end{array}$ \\
\hline SFHo & 0.1583 & 16.19 & 245.4 & -467.8 & 31.57 & 47.10 & 0.7609 & 0.7606 & 11.88 & 2.059 & 2.27 \\
\hline SFHx & 0.1602 & 16.16 & 238.8 & -457.2 & 28.67 & 23.18 & 0.7179 & 0.7174 & 11.97 & 2.130 & 2.36 \\
\hline STOS(TM1) & 0.1452 & 16.26 & 281.2 & -285.3 & 36.89 & 110.79 & 0.6344 & 0.6344 & 14.56 & 2.23 & 2.62 \\
\hline HS(TM1) & 0.1455 & 16.31 & 281.6 & -286.5 & 36.95 & 110.99 & 0.6343 & 0.6338 & 13.84 & 2.21 & 2.59 \\
\hline HS(TMA) & 0.1472 & 16.03 & 318.2 & -572.2 & 30.66 & 90.14 & 0.6352 & 0.6347 & 14.44 & 2.02 & 2.48 \\
\hline HS(FSUgold) & 0.1482 & 16.27 & 229.5 & -523.9 & 32.56 & 60.43 & 0.6107 & 0.6102 & 12.52 & 1.74 & 2.34 \\
\hline $\mathrm{LS}(180)$ & 0.1550 & 16.00 & 180.0 & -450.7 & 28.61 & 73.82 & 1 & 1 & 12.16 & 1.84 & 2.02 \\
\hline $\mathrm{LS}(220)$ & 0.1550 & 16.00 & 220.0 & -411.2 & 28.61 & 73.82 & 1 & 1 & 12.62 & 2.06 & 2.14 \\
\hline
\end{tabular}

TABLE 3

Properties of NUCLEI FOR SFHo AND SFHx

\begin{tabular}{ccccc}
\hline \hline & $\begin{array}{c}\mathrm{E}\left({ }^{208} \mathrm{~Pb}\right) \\
{[\mathrm{MeV}]}\end{array}$ & $\begin{array}{c}\mathrm{R}\left({ }^{208} \mathrm{~Pb}\right) \\
{[\mathrm{fm}]}\end{array}$ & $\begin{array}{c}\mathrm{E}\left({ }^{90} \mathrm{Zr}\right) \\
{[\mathrm{MeV}]}\end{array}$ & $\begin{array}{c}\mathrm{R}\left({ }^{90} \mathrm{Zr}\right) \\
{[\mathrm{fm}]}\end{array}$ \\
\hline SFHo & -7.76 & 5.44 & -8.60 & 4.19 \\
SFHx & -7.87 & 5.41 & -8.55 & 4.19 \\
\hline \hline
\end{tabular}

and high-density parts of the isospin sector separately. There are 17 parameters including: the scalar-isoscalar meson mass $m_{\sigma}$, the standard non-linear Walecka model couplings $g_{\sigma}, g_{\omega}, g_{\rho}, b$, and $c$, the two fourth-order vector meson couplings $\zeta$ and $\xi$, and the parameters $a_{1-6}$ and $b_{1-3}$ which control the symmetry energy as a function of density. These parameters are varied to ensure that both EOSs have saturation properties which agree with that predicted from nuclear masses and giant monopole resonances. In addition, both predict binding energies and charge radii for ${ }^{208} \mathrm{~Pb}$ and ${ }^{90} \mathrm{Zr}$ that are within $2 \%$ of the experimental values. We ensure that the pressure of neutron matter is always positive and always increases as a function of the density. We ensure that the maximum mass is larger than 1.93 solar masses (the lower $1-\sigma$ limit from Demorest et al. (2010)). We compare our results to LS(180) and FSUGold even though these are inconsistent with this maximum mass because they are still commonly used in the supernova community. The requirement that the speed of sound is not superluminal is automatically enforced in this fully covariant model. In our baseline model, SFHo, we also fit the most probable mass-radius curve from Steiner et al. (2010), and in our extreme model, SFHx, we attempt to minimize the radius of low-mass neutron stars yet remaining consistent with the other constraints given above. This forces the value of $L$ for SFHx to be on the lower edge of the typical range of $20-120 \mathrm{MeV}$. We always take the mass of the neutron to be $939.565346 \mathrm{MeV}$, the mass of the proton to be $938.272013 \mathrm{MeV}$, the mass of the $\omega$ meson to be 762.5 $\mathrm{MeV}$, the mass of the $\rho$ meson to be $770 \mathrm{MeV}$, and we use $\hbar c=197.3269631 \mathrm{MeV}$ fm (Mohr et al. 2008). The full parameter list for both models is given in Table 1 The saturation properties, nuclear binding energies and nuclear charge radii for our models are given in Tables 2 and 3. We remark that the values given in Table 2 can be slightly different to previously published ones, e.g. by Hempel et al. (2011), due to a different treatment of the nucleon rest masses. In the present work we are using the measured masses from above for all of the HS EOS, to obtain the correct low-density limit. This treatment also leads to a slight splitting of the neutron and proton

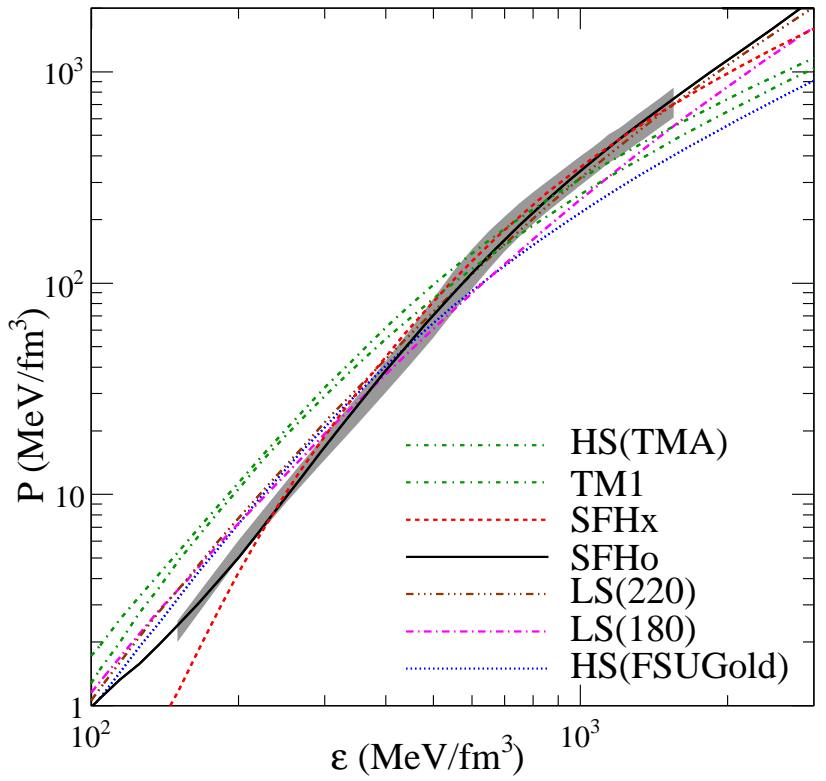

FiG. 1.- Pressure of zero temperature beta-equilibrium matter as a function of the total energy density. The gray region gives the $1 \sigma$ confidence limits on the EOS from Steiner et al. (2010). The curve labeled TM1 applies for both the HS(TM1) and STOS EOSs.

effective masses, $m_{n}^{*}$ and $m_{p}^{*}$ respectively. The value of $J$ for LS in Table 2 differs from the published value by Lattimer \& Swesty (1991) of $29.3 \mathrm{MeV}$. These authors computed $J$ as the energy difference between neutron and nuclear matter whereas we are calculating $J$ as the second derivative with respect to $Y_{e}$ at the saturation point. In Table 2, we also give the saturation density $n_{B}^{0}$, the binding energy of nuclear matter $E_{0}$, the skewness of nuclear matter $K^{\prime}$, the reduced neutron and proton effective masses at saturation, the radii of 1.4 solar mass neutron stars at $T=0$, the maximum mass at $T=0$, and the maximum mass for stars with constant entropy $s=4$ in beta-equilibrium without neutrinos. The $T=0$ EOSs for all of the models constructed with nucleon degrees of freedom are given in Figure 1], along with the constraints from Steiner et al. (2010). Figure 2 gives the corresponding mass-versus radius curves and also the constraints from Steiner et al. (2010).

For densities below saturation density we apply the statistical model from Hempel \& Schaffner-Bielich (2010) (HS) for the description of non-uniform nuclear matter in nuclear statistical equilibrium (NSE), i.e. for the forma- 


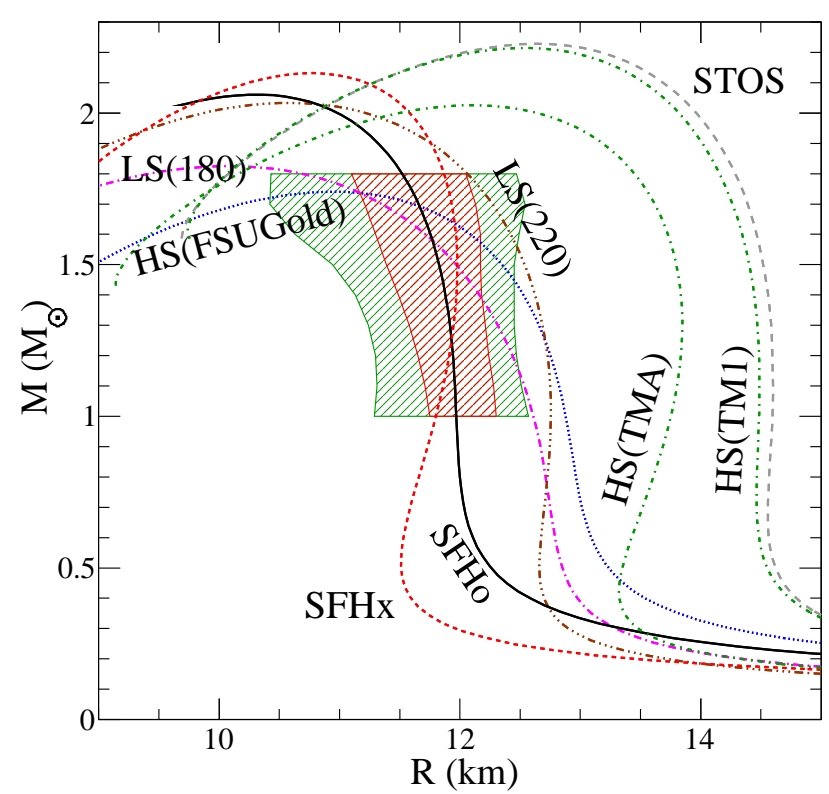

FIG. 2.- Neutron star mass-radius curve for modern supernova equations of state. The red (green) region outlines the one (two) $\sigma$ confidence limits from Steiner et al. (2010).

tion of light and heavy nuclei within the gas of unbound nucleons. For the unbound nucleons, we utilize the SFHo and SFHx relativistic mean-field interactions. At low densities, the description of nuclei is based on measured experimental binding energies (Audi et al. 2003), which are combined with theoretical nuclear structure calculations for exotic nuclei without measured data. Here the finite range droplet model of Möller et al. (1995) was chosen because of its excellent reproduction of experimental binding energies, with a rms deviation of only $0.669 \mathrm{MeV}$. Due to the use of nuclear structure data, shell effects are automatically included. HS goes beyond the single nucleus approximation and utilizes a distribution of different nuclear species, and the results for light nuclei are in agreement with more sophisticated quantum many-body models (Hempel et al. 2011). Also, the recent experimental study of Qin et al. (2012) indicates that the HS model is well suited for the description of matter at finite temperature and densities around a few tenths of saturation density. At even larger densities in the HS model the disappearance of nuclei and smooth transition to uniform nuclear matter is assured by an excluded volume description. Finally we calculate the EOS in tabular form, covering densities from $10^{-12}$ to 10 $\mathrm{fm}^{-3}$, temperatures from 0.1 to $160 \mathrm{MeV}$, and electron fractions from 0 to 0.6 , including detailed information about the nuclear composition and the thermodynamic properties. The tables are suitable for use in astrophysical simulations and are available online $\square$

The resulting EOS will be compared below with the LS EOS, with the different compressibilities $180 \mathrm{MeV}$ (LS180), $220 \mathrm{MeV}$ (LS220), and $375 \mathrm{MeV}$ (LS375), and with STOS. Moreover, we will also include into our comparison results obtained using the quark-hadron hybrid EOS from Fischer et al. (2011). We select the model with

1 See http://phys-merger.physik.unibas.ch/ hempel/eos.html. bag constant $B^{1 / 4}=155 \mathrm{MeV}$ and including corrections from the strong coupling constant, $\alpha_{S}=0.3$ (hereafter QB155 $\left.\alpha_{S} 03\right)$, where the phase transition to quark matter takes place at nuclear saturation density for temperatures around $10 \mathrm{MeV}$ and $Y_{e} \simeq 0.3$ (for details, see Fischer et al. 2011). The hadronic part of this EOS table is based on the STOS EOS, and these two EOS are identical at sub-saturation densities where quarks are not present.

\section{CORE-COLLAPE SUPERNOVA SIMULATIONS}

In this section, we will compare results from $\mathrm{SN}$ simulations obtained using the SFHo EOS with the standard EOS LS180 and the two TM1 RMF parameterizations STOS and HS. Furthermore, we will also compare SFHo with the hybrid EOS QB $155 \alpha_{S} 03$, for which explosions were obtained recently even in spherically symmetric simulations (Fischer et al. 2011).

TABLE 4

NEUTRINO REACTIONS CONSIDERED INCLUDING REFERENCES.

\begin{tabular}{|c|c|}
\hline Reaction $^{\mathrm{a}}$ & References \\
\hline$\nu_{e}+n \rightarrow p+e^{-}$ & Bruenn (1985) \\
\hline $\bar{\nu}_{e}+p \rightarrow n+e^{+}$ & Bruenn (1985) \\
\hline$\nu_{e}+(A, Z-1) \rightarrow(A, Z)+e^{-}$ & Langanke et al. (2003), \\
\hline & Hix et al. (2003) \\
\hline$\nu+N \rightarrow \nu^{\prime}+N$ & Bruenn (1985) \\
\hline$\nu+(A, Z) \rightarrow \nu^{\prime}+(A, Z)$ & Bruenn (1985) \\
\hline$\nu+e^{ \pm} \rightarrow \nu^{\prime}+e^{ \pm}$ & Bruenn (1985), \\
\hline & $\begin{array}{l}\text { Mezzacappa \& Bruenn } \\
\text { Mezzacappa \& Bruenn } \\
(1993 a)\end{array}$ \\
\hline$\nu+\bar{\nu} \rightarrow e^{-}+e^{+}$ & Bruenn (1985) \\
\hline & Mezzacappa \& Messer (1999) \\
\hline$\nu+\bar{\nu}+N+N \rightarrow N+N$ & Hannestad \& Raffelt (1998) \\
\hline$\nu_{e}+\bar{\nu}_{e} \rightarrow \nu_{\mu / \tau}+\bar{\nu}_{\mu / \tau}$ & Buras et al. (2003) \\
\hline
\end{tabular}

\subsection{Supernova model}

Our core-collapse SN model, AGILE-BOLTZTRAN, is based on general relativistic radiation hydrodynamics in spherical symmetry. It employs three-flavor Boltzmann neutrino transport (see Liebendoerfer et al. 2004, and references therein). We use the standard weak processes following Bruenn (1985), see Table 4 for details. In addition, we include the improved rates for electroncaptures on heavy nuclei from Langanke et al. (2003) and Hix et al. (2003), weak magnetism and nucleon recoil based on Horowitz (2002), and the annihilation of trapped electron neutrino pairs has been implemented in Fischer et al. (2009) following Buras et al. (2003).

For NSE conditions $(T>0.45 \mathrm{MeV})$, we implement the baryon EOS tables specified above. For non-NSE, we assume the ideal gas of ${ }^{28} \mathrm{Si}$ for the baryon EOS. On top of the baryons, also for NSE, contributions from electrons, positrons and photons are added to the EOS using Timmes \& Arnett (1999). Recently, this Si-gas approximation has been replaced by a nuclear reaction network, based on the nuclear composition given by the progenitor model. It allows, e.g., for a smooth NSE-to-non-NSE transition as well as to simulate a large domain of the progenitor star (for details, see Fischer et al. 2010).

The simulations we will discuss further below are launched from iron-core progenitors. We use the 
$15 \mathrm{M}_{\odot}$ model from Woosley et al. (2002) for regular core-collapse supernovae and the $40 \quad \mathrm{M}_{\odot}$ from Woosley \& Weaver (1995) for the black-hole formation scenario. None of the spherically symmetric simulations using the purely hadronic EOS results in an explosion for the considered simulation times.

\subsection{Simulation results}

A detailed supernova EOS comparison study for LS180 and STOS as well as several HS EOS tables with different RMF parameterizations has been published very recently in Hempel et al. (2012). Here, we extend their analysis and include in addition $\mathrm{SFHo} / \mathrm{SFHx}$.

During the core-collapse phase, the composition is dominated by heavy nuclei. At low temperatures in nonNSE the EOS based on the ideal gas of Si-nuclei has been applied in all simulations except for HS(TM1) where we used our simplified nuclear reaction network. Hence, all EOS lead to very similar structures in the simulations at low temperatures, see e.g. the equal entropy per baryon profiles in the outer layers $\left(M_{\mathrm{B}}>1.5 \mathrm{M}_{\odot}\right)$ as illustrated in Fig. 3 (except HS(TM1)). Note also that LS(180) has a different non-NSE treatment based on the ideal Si-gas. Moreover, structure differences arise at higher temperatures in NSE, which will be discussed $\mathrm{i}+\mathrm{n}$ the following paragraph. In general, low-density differences (i.e. on top of the bounce shock $0.7<M_{\mathrm{B}}<1.5 \mathrm{M}_{\odot}$ in Fig. 3 between STOS and HS(TM1) are related to a different description of heavy nuclei. The statistical approach of HS is largely based on experimentally known masses including shell effects. It resembles the ideal gas of ${ }^{56} \mathrm{Ni} /{ }^{56} \mathrm{Fe}$ at the transition to non-NSE by construction. In contrast, the Thomas-Fermi approximation of STOS gives heavy nuclei which are too strongly bound and does not perform well at this transition. Hempel et al. (2012) explained how these differences affect the $Y_{e}$ evolution. Furthermore, entropy differences between STOS and HS(TM1) originate from the missing kinetic entropy contribution of heavy nuclei in STOS. Since we use the same low-density description for nuclei as in HS, simulations using SFHo and SFHx result in the same conditions during the core-collapse phase. The simulation results for QB155 $\alpha_{S} 03$ are similar to STOS at low densities where quarks are not present. Even though they are based on the same low-density EOS, small differences have emerged because the core has already entered the quark-hadron mixed phase.

Only a few tens of milliseconds before core bounce, nuclear saturation density is reached at the center. At these conditions high-density EOS differences become large which in turn lead to different dynamical evolutions. We define the moment of core bounce when the central density reaches its maximum before shock breakout. The EOS with highest central density, LS(180), results in the smallest enclosed baryon mass (see Fig. 31). The two TM1 EOSs, STOS and HS(TM1), result in the lowest central densities. Differences between STOS and HS(TM1) are due to the different description of heavy nuclei, as already discussed above. Furthermore, the presence of light nuclei, which are taken into account in HS, also has an impact on thermodynamic properties. Comparing STOS and QB155 $\alpha_{S} 03$, the most pronounced differences arise above nuclear saturation densities which are due to the presence of deconfined quarks (in partic-

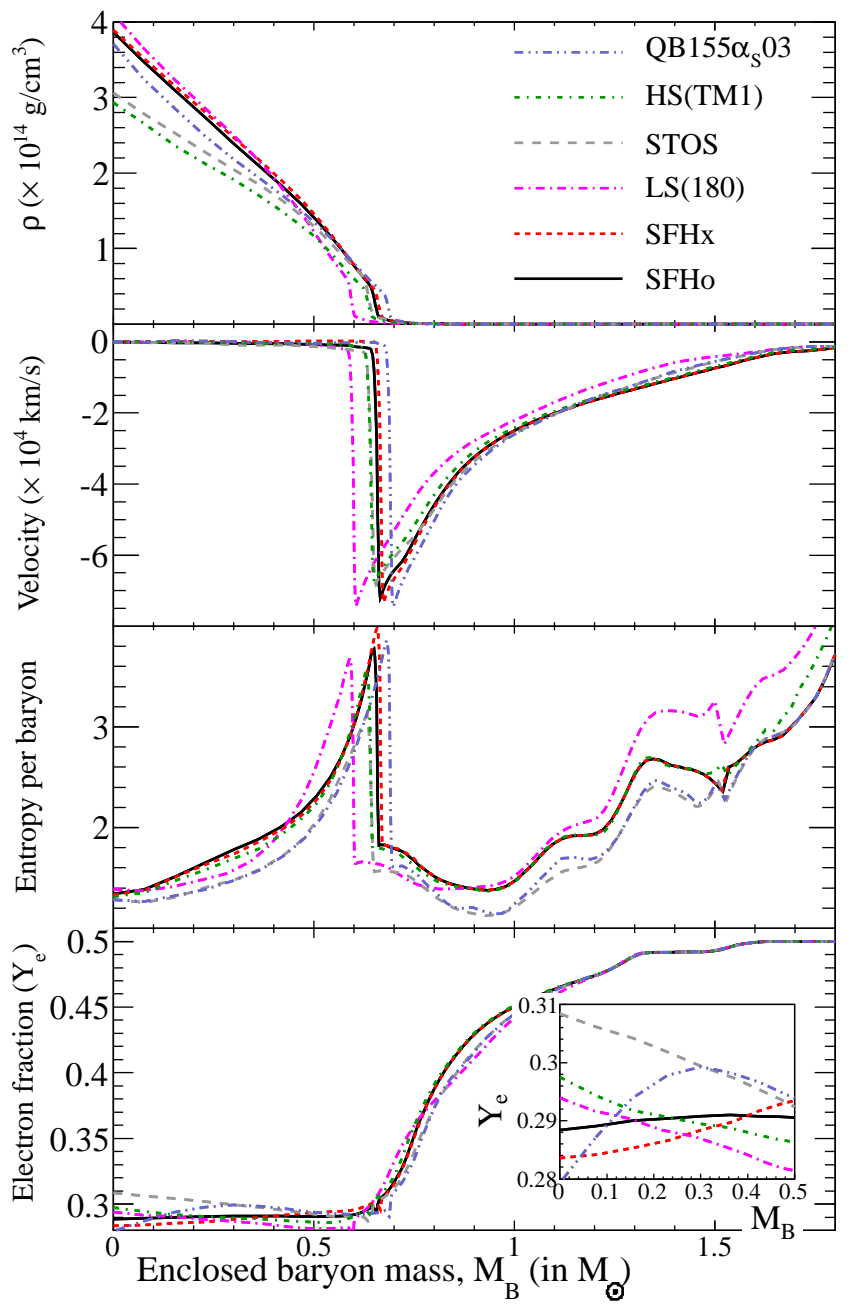

FIG. 3.- Bounce profiles of selected quantities for the $15 \mathrm{M}_{\odot}$ models, comparing the different EOS under investigation.

ular s-quarks). They soften the high-density EOS and result in higher central densities and significantly lower central $Y_{e}$ at core bounce (for details, see Fischer et al. 2011).

One of our new models, SFHx, gives the lowest electron fractions for $M<0.25 \mathrm{M}_{\odot}$, due to its low slope of the symmetry energy $L$. Compared with LS(180), SFHo and SFHx reach similarly high central densities and low central $Y_{e}$ at core bounce (see Fig. 3 and further discussion below). Note that LS(180) has lowest incompressibility $K$ and symmetry energy $J$ among all EOS under consideration. However, the baryon mass enclosed inside the bounce shock for the two new EOS differs by about $0.1 \mathrm{M}_{\odot}$ and is actually more similar to the TM1 results, which has an extremely high $K$ and $J$. These results provide no clear correlations between individual nuclear matter properties of the EOS (see Table 3) and the different conditions, e.g., central density and $Y_{e}$ obtained at core bounce for these EOS (see Fig. 3). It seems difficult to disentangle individual nuclear EOS properties from the different conditions obtained in simulations of stellar core collapse.

The central object formed at core bounce, i.e. the protoneutron star (PNS), is hot and lepton rich in which sense it differs from the final remnant neutron star. After 
bounce, the shock starts to propagate outwards with initially positive velocities. Simultaneously, mass accretion from the outer part of the stellar core continuously grows the mass of the PNS. Moreover, the expanding bounce shock loses energy due to heavy-nuclei dissociation and neutrino emission. The neutrino emission is related to a large number of electron captures on protons during the shock passage across the neutrinospheres. It releases a burst of $\nu_{e}$ of several $10^{53} \mathrm{erg} \mathrm{s}^{-1}$ for a short timescale between 5-20 ms after core bounce. Both sources of energy loss turn the expanding and dynamic (i.e. accompanied with matter outflow) bounce shock into an accretion front, the standing accretion shock (SAS), already between $5-10 \mathrm{~ms}$ after core bounce. The later PNS evolution is determined from mass accretion and the subsequent PNS compression which leads to continuously rising central density and temperature. For a given mass accretion rate, determined from the progenitor, and otherwise identical simulation setup, the timescale for the PNS compression is directly related to the EOS. On timescales on the order of several $100 \mathrm{~ms}$, EOS differences lead to different neutrinospheres and shock radii, displayed in Fig. 4. The neutrinospheres can be used to characterize the size of the central protoneutron star as they are located in its outer envelope.

At $100 \mathrm{~ms}$ post bounce, LS(180) has the smallest shock radius at $130 \mathrm{~km}$ because the shock had lower energies at bounce. However, its contraction proceeds initially, up to about $200 \mathrm{~ms}$ post bounce, somewhat slower than those for the EOS HS(TM1) and SFHo/SFHx. This behavior is related to the rapid PNS contraction obtained for the latter. By comparing with STOS, one can identify the effect of the different treatment of heavy nuclei and in addition the consistent inclusion of light nuclei for HS and also for SFHo/SFHx (for details, see Hempel et al. 2012). These differences of the low-density EOS result in an initially accelerated PNS contraction of HS(TM1). STOS leads to the generally slowest PNS contraction and hence slowest shock contraction as well. Differences between STOS and QB155 $\alpha_{S} 03$ are related to the presence of quarks, in particular strange quarks, at and above saturation densities. These become abundant during the PNS contraction as the PNS domain occupied by quark matter grows continuously during the accretion phase (for details about the scenario, see Fischer et al. 2011). The high-density EOS is dominated by the quark matter description after about $200 \mathrm{~ms}$ post bounce. This results in an accelerated contraction of the PNS for QB155 $\alpha_{S} 03$ in comparison to STOS (see Fig. 4). Note that at about $300 \mathrm{~ms}$ post bounce, the PNS becomes gravitationally unstable and collapses. A massive quark core forms at the PNS center, which causes the collapse to halt and an additional shock wave forms at saturation density. The subsequent shock expansion and even acceleration, accompanied by mass outflow, determines the onset of explosion for this model. Since none of the purely hadronic EOSs lead to an explosion, we only show and discuss results up to the moment of PNS collapse for QB155 $\alpha_{S} 03$.

It is difficult to predict astrophysical simulation results from only nuclear matter properties at saturation density and zero temperature. For example both HS(TM1) and STOS are based on the same nucleon interactions TM1 (see Table 31). However, the additional inclusion of light nuclei and the different description of heavy nuclei, rel-

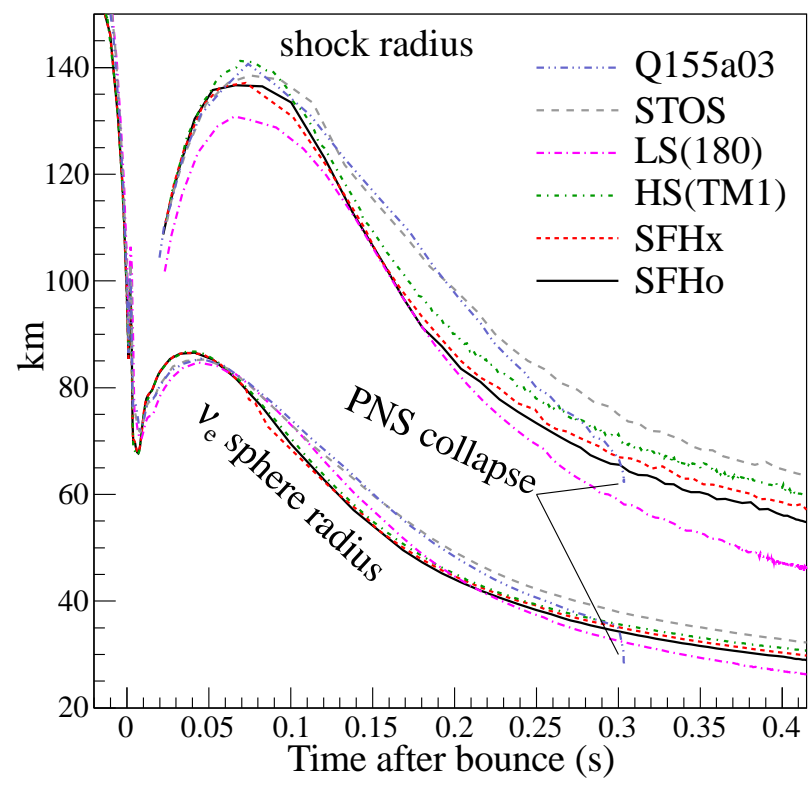

FIG. 4.- Evolution of the shock radii (thick lines) and $\nu_{e}$-spheres (thin lines) for the $15 \mathrm{M}_{\odot}$ models, comparing the different EOS under investigation.

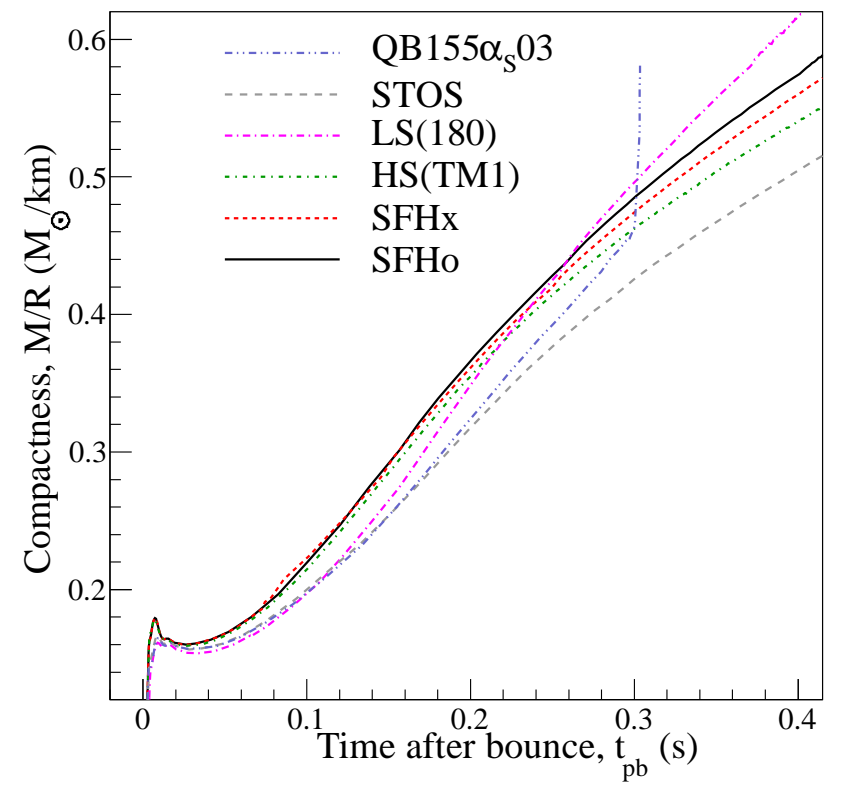

FIG. 5.- The compactness of the protoneutron star after bounce, defined by the gravitational enclosed mass and radius of the electron neutrinosphere.

evant for the low- and intermediate-density EOS have a significant impact on the conditions at bounce and then also on the later PNS structure and contraction behavior. The early PNS evolution for our new parameterizations SFHo and SFHx, up to about $50 \mathrm{~ms}$ post bounce, resembles those of HS(TM1) (see the neutrinospheres in Fig. 4). Later, the PNS contraction proceeds even faster than for HS(TM1), reflecting the softer high-density EOS of SFHo/SFHx. On timescales of several $100 \mathrm{~ms}$, the PNS and shock contractions slow down for all the RMF EOSs as a consequence of the continuously growing en- 
closed mass. This effect is significantly weaker for the non-relativistic LS(180) EOS.

Fig. 5 shows the evolution of the compactness, the ratio of the enclosed gravitational mass to the radius. We evaluate these quantities at the electron neutrinosphere, representative of the protoneutron star surface. The EOSs which result in the most compact protoneutron stars at core bounce and few tens of milliseconds after are those of SFHo and SFHx, closely followed by HS(TM1). At core bounce and up to $50 \mathrm{~ms}$ post bounce, LS180 results in the least compact protoneutron star. However, during the later post-bounce evolution for LS180 the compactness of the central protoneutron star grows fastest among all of the EOSs under exploration. For HS(TM1) the protoneutron star contraction proceeds on the same timescale as STOS, only on a slightly more compact magnitude. This can be attributed to the more compact, configuration at core bounce for HS(TM1). STOS and QB155 $\alpha_{S} 03$ proceed along identical lines until about $100 \mathrm{~ms}$ post bounce, when quark matter begins to dominate the protoneutron star evolution. Note, at that moment a quark-hadron phase transition to strange quark matter has not been fully achieved, i.e. most mass inside the protoneutron star is only in the quark-hadron mixed phase. The EOSs with most compact protoneutron stars at bounce and during the early post-bounce evolution are SFHo and SFHx which remain the most compact ones until about $300 \mathrm{~ms}$ post bounce, when only LS180 becomes more compact. Up to this moment, SFHo and SFHx have the same compactness, and differences observed during the later evolution remain small.

In addition to the simulations using the $15 \mathrm{M}_{\odot}$ progenitor, we also include the more massive $40 \mathrm{M}_{\odot}$ progenitor from Woosley et al. (2002) into our EOS comparison in supernova simulations. This progenitor has been discussed before within the scenario of black-hole formation, also explored for the LS and STOS supernova EOSs (see, e.g., Sumivoshi et al. 2006, 2007; Nakazato et al. 2008; Fischer et al.|2009; O'Connor \& Ott|2011; Hempel et al. 2012). The moment of black hole formation is defined, within our general relativistic framework (for details, see Liebendoerfer et al. 2004), as the central lapse function approaches zero and stable numerical solutions for the evolution equations cannot be obtained anymore. The lapse function determines, e.g., time dilatations and gravitational Doppler shift effects. Note that corecollapse events which result in a black hole instead of an explosion are as bright as normal supernova explosions with respect to neutrino signal. The possible future detection of such a neutrino signal from a Galactic event may be used to further constrain the high-density EOS, see Nakazato et al. (2010).

The moment of black-hole formation is approached during the post-bounce evolution, during which the mass enclosed inside the PNS grows continuously in the absence of an explosion. The timescale for the central density (and also temperature) rise is given by the PNS contraction behavior, which in turn depends on the nuclear EOS. We list selected properties at the onset of PNS collapse in Table 5 for all hadronic EOS under investigation.

The PNS compression can be illustrated best via the central density evolution, shown in Fig. 6. comparing the different hadronic EOSs under investigation. The values of the central density cluster around only two different

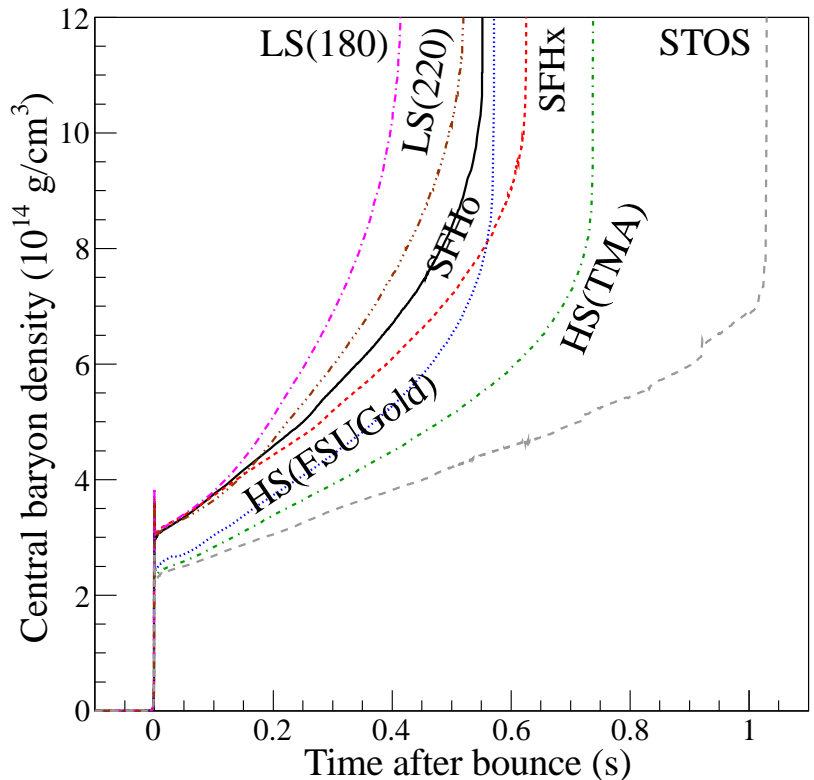

FIG. 6.- Central density evolution for the $40 \mathrm{M}_{\odot}$ model, comparing the hadronic EOS under investigation.

values, $3.2 \times 10^{14}$ and $2.5 \times 10^{14} \mathrm{~g} / \mathrm{cm}^{3}$, immediately after the bounce. It is likely that this is related to the properties of the EOS at low-density, and thus also to the radii of very low-mass neutron stars. A similar (but not exact) clustering is observed in the lower-right hand corner of Fig. 2 where STOS, HS(TM1), and HS(FSUGold) have larger radii for 0.4 solar mass neutron stars. This grouping lasts only for $300 \mathrm{~ms}$ after which time the EOS at higher densities becomes important.

HS(TMA) has a higher incompressibility but lower symmetry energy than STOS (which uses TM1) and leads to a significantly shorter (about $300 \mathrm{~ms}$ ) accretion time until black-hole formation (see Fig. 6). This would indicate that the symmetry energy plays the dominant role. However, SFHo and SFHx do not obey this trend. Even though SFHo has a higher incompressibility and higher symmetry energy than SFHx, it leads to a shorter (about $50 \mathrm{~ms}$ ) accretion time until black-hole formation. Instead of using nuclear matter properties, one could expect that our results could be explained by the maximum mass of cold neutron stars, which is an integrated quantity of the EOS. However, by comparing the times until black hole formation from Table 5) with the numbers in Table 2, it turns out that there is no monotonic correlation. For example the collapse of FSUgold occurs about $160 \mathrm{~ms}$ later than for LS(180), even though the maximum mass of $\mathrm{LS}(180)$ is $0.1 \mathrm{M}_{\odot}$ larger.

Note that the two RMF EOS parameterizations, TM1 and TMA, fail to fulfill both zero temperature maximum mass and radius constraints, while $\mathrm{LS}(220)$ is on the edge of acceptance, see Fig. 2. The RMF EOS based on TM1 and TMA result in sufficiently high maximum masses but give at the same time radii which are too large. With the black-hole formation scenario explored here, these large radii are due to slower PNS contraction which results in an extended post-bounce mass accretion period until black-hole formation (see Fig. 6). On the other hand, FSUgold results in reasonably small neutron 


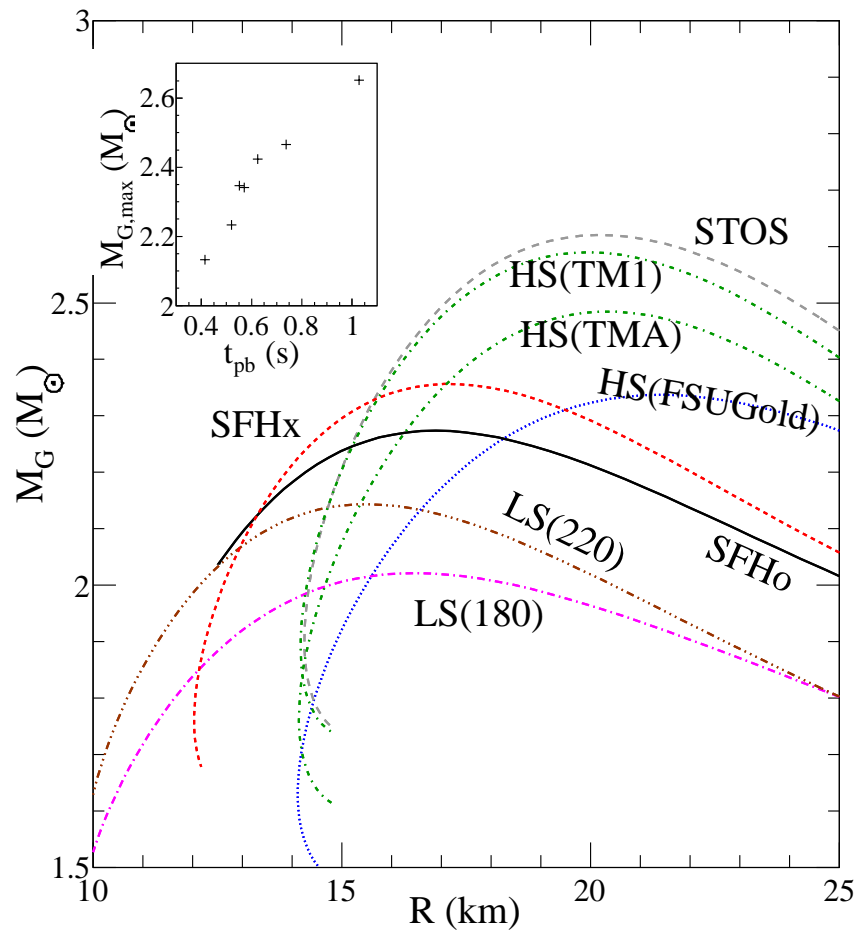

FIG. 7.- Mass-radius relations at constant entropy per baryon of $4 \mathrm{k}_{B}$ comparing the hadronic EOSs under investigation.

TABLE 5

Selected QuANTities at THE ONSET OF PNS COllapse AND BLACK-HOLE FORMATION.

\begin{tabular}{lccccc}
\hline \hline EOS & $\begin{array}{c}t_{p b}{ }^{\mathrm{a}} \\
{[s]}\end{array}$ & $\begin{array}{c}\rho^{\mathrm{b}} \\
{\left[10^{15} \mathrm{~g} / \mathrm{cm}^{3}\right]}\end{array}$ & $\begin{array}{c}T^{\mathrm{c}} \\
{[\mathrm{MeV}]}\end{array}$ & $\begin{array}{c}M_{B}{ }^{\mathrm{d}} \\
{\left[\mathrm{M}_{\odot}\right]}\end{array}$ & $\begin{array}{c}M_{G}{ }^{\mathrm{e}} \\
{\left[\mathrm{M}_{\odot}\right]}\end{array}$ \\
\hline LS(180) & 0.415 & 1.292 & 29.978 & 2.227 & 2.133 \\
LS(220) & 0.521 & 1.324 & 31.446 & 2.350 & 2.233 \\
SFHo & 0.551 & 1.067 & 46.691 & 2.477 & 2.347 \\
HS(FSUgold) & 0.571 & 1.058 & 48.104 & 2.465 & 2.341 \\
SFHx & 0.625 & 0.803 & 40.830 & 2.552 & 2.424 \\
HS(TMA) & 0.737 & 0.943 & 46.708 & 2.626 & 2.466 \\
STOS & 1.028 & 0.769 & 49.705 & 2.864 & 2.652 \\
\hline \hline
\end{tabular}

Notes:

atime post bounce

${ }^{\mathrm{b}}$ central baryon density

${ }^{\mathrm{C}}$ central temperature

denclosed baryon mass

enclosed gravitational mass

star radii but fails to fulfill the current maximum zero temperature mass constrain of $1.97 \pm 0.04 \mathrm{M}_{\odot}$. It leads to a significantly shorter time until black-hole formation than for TMA and TM1, comparable to SFHo which has a larger incompressibility but slightly lower symmetry energy than FSUgold. On the other hand, SFHo and SFHx fulfill both mass and radius constraints by construction. It is interesting that the models which fulfill the mass-radius and maximum mass constraints, i.e. LS(220), SFHo, and SFHx, give rather similar results for $t_{B H}$, which seems to be constrained to $500-650 \mathrm{~ms}$. It appears that neutron star radius measurements constrain the time to black hole formation for $40 M_{\odot}$ progenitors.

Hempel et al. (2012) demonstrated that maximum neutron star mass determined from the EOS in betaequilibrium at $s=4 \mathrm{k}_{B}$ /baryon was strongly correlated with the time until black-hole formation. This time can be measured with currently available neutrino detectors given a galactic core-collapse supernova. The associated mass versus radius curves and their relationship with the time to black hole formation is displayed in Fig. 7 for the hadronic EOSs under investigation. The corresponding values are also listed in the last column of Table 2. Note that the maximum masses increase for all EOS compared to the $T=0$ case. (compare also Figs. 7 and 2). Pressure is more sensitive than the energy density to the temperature effects because of the large nucleon mass and thus larger temperatures in increase the maximum mass. If we compare the maximum masses of the $s=4 \mathrm{k}_{B}$ /baryon configurations from Table 2 with the time until blackhole formation from Table 5. we find that these quantities have the same ordering, i.e. show an (almost) strictly monotonic correlation.

\section{SUMMARY AND CONCLUSIONS}

We developed two new relativistic mean-field (RMF) interactions, based on which we constructed the two new supernova EOSs, SFHo and SFHx, in such a way to fulfill current constraints from neutron star mass and radius measurements. Moreover, the new EOSs are consistent with nuclear experimental constraints on matter near and below the saturation density. These new EOSs provide more variation in the set of EOS tables which can be used by the core-collapse supernova community instead of EOSs based on TMA or TM1 which are now ruled out by observations.

The new EOS were implemented in core-collapse supernova simulations of massive iron-core progenitors. We compared the results with the commonly used nonrelativistic EOS of Lattimer \& Swesty (1991) and the RMF EOS of Shen et al. (1998). Moreover, we include into our EOS comparison also the recently introduced quark-hadron hybrid EOS from Fischer et al. (2011) and the RMF EOS from Hempel \& Schaffner-Bielich (2010) based on the parameterizations TM1, TMA and FSUgold. We compared the different EOS during the ironcore collapse phase, which is dominated by heavy nuclei, and confirmed already reported differences between these EOS (see, e.g., Hempel et al. 2012). We extend the analysis and include SFHo/SFHx. Differences become large only slightly before and after core bounce, when the central density exceeds normal nuclear matter density. The post-bounce mass accretion phase is ideal to study the protoneutron star contraction behavior, which reflects the EOS underlying nuclear matter properties for a given progenitor choice. We found that the two new EOS which give small radii for cold neutron stars also lead to the most compact protoneutron stars in the first $300 \mathrm{~ms}$ after bounce. However, it is not easy to disentangle the relationship between individual nuclear matter properties, given at zero temperature near the saturation density, and the outcome of our supernova simulations.

We also find the EOS classifications 'soft' or 'stiff' misleading. Implicitly what is meant by soft or stiff is that the EOS has a lower or higher pressure. However, corecollapse supernova explore a large range of densities and temperatures, and an EOS which has a higher pressure at one density and temperature may have a lower pressure at another density and temperature. This is particularly evident with the black-hole formation time as described 
here and in Hempel et al. (2012), as EOSs like SFHx which have a low pressure at zero temperatures near the saturation density, have a larger time until black hole formation than the other EOSs because their pressure at $s=4$ is larger. The addition of quark degrees of freedom only further complicates this issue.

Moreover, we explored the possible correlation between the EOS and outcome of the black-hole formation scenario in the absence of a supernova explosion. We confirm the analysis of Hempel et al. (2012), where only a correlation between finite entropy per baryon configurations of static protoneutron stars and the time until black-hole formation was found. Note that the neutrino signals of these events are as bright as ordinary supernova explosions. But at the moment of black-hole formation the signal suddenly stops which makes this time measurable with neutrino telescopes. The possible future neutrino observation of such a Galactic event will constrain the high-density and finite-entropy EOS significantly, complementary to future neutron star mass and radius observations.

One conclusion from this study is that it seems to be difficult to understand the effect of the EOS in corecollapse supernovae by analyzing non-exploding models. To tackle the impact of the EOS on the explosion mechanism, multi-dimensional simulations are necessary, see e.g. Marek et al. (2009). Furthermore, it remains to be explored how different nuclear EOS can influence longterm cooling to protoneutron stars after the supernova explosion has been launched. Therefore, the implementation of weak processes consistent with the nuclear EOS is required (see, e.g., Reddy et al. 1998, 1999). This may impact the neutrino cooling timescale and also the extent of protoneutron star convection as studies recently described by Roberts et al. (2012). The nuclear EOS may also be important for the proton-to-baryon ratio of the material ejected form the protoneutron star surface known as neutrino-driven wind relevant for the nucleosynthesis of heavy elements. Moreover, a possible EOS impact within the cooling of protoneutron stars on the emitted neutrino signal may also be of relevance for neutrino oscillation studies, in particular those which explore collective phenomena in the presence of large neutrino but small matter densities.

\section{ACKNOWLEDGEMENTS}

The authors would like to thank S. Reddy, L. Roberts and I. Sagert for several discussions on this work. The supernova simulations were performed at the computer center of the Helmholtzzentrum für Schwerionenforschung GmbH (GSI) in Darmstadt, Germany. A.W.S. was supported by DOE grant DE-FG02-00ER41132. M.H. and T.F. are grateful for support from CompStar, a research networking program of the ESF. M.H. acknowledges support from the High Performance and High Productivity Computing (HP2C) project, the Swiss National Science Foundation (SNF) under project number no. 200020-132816/1, and ENSAR/THEXO. T.F. is supported by the Swiss National Science Foundation under project no. PBBSP2-133378 and HIC for FAIR.

\section{REFERENCES}

Audi, G., Wapstra, A. H., \& Thibault, C. 2003, Nucl. Phys. A, 729,337

Baron, E., Cooperstein, J., \& Kahana, S. 1985, Phys. Rev. Lett., 55,126

Bethe, H. A., \& Wilson, James, R. 1985, ApJ, 295, 14

Bruenn, S. W. 1985, ApJS, 58, 771

Buras, R., Janka, H.-T., Keil, M. T., Raffelt, G. G., \& Rampp, M. 2003, ApJ, 587, 320

Burrows, A., Livne, E., Dessart, L., Ott, C., \& Murphy, J. 2006, ApJ, 640, 878

Colo, G., Van Giai, N., Meyer, J., Bennaceur, K., \& Bonche, P. 2004, Phys. Rev. C, 70, 024307

Demorest, P. B., Pennucci, T., Ransom, S. M., Roberts, M. S. E., \& Hessels, J. W. T. 2010, Nature, 467, 1081

Fischer, T., Whitehouse, S., Mezzacappa, A., Thielemann, F.-K., \& Liebendorfer, M. 2010, Astron.Astrophys., 517, A80

Fischer, T., Whitehouse, S. C., Mezzacappa, A., Thielemann, F.-K., \& Liebendörfer, M. 2009, Astronom. Astrophys., 499, 1

Fischer, T., Sagert, I., Pagliara, G., et al. 2011, ApJS, 194, 39

Hannestad, S., \& Raffelt, G. 1998, Astrophys.J., 507, 339

Hebeler, K., Lattimer, J. M., Pethick, C. J., \& Schwenk, A. 2010 Phys. Rev. Lett., 105, 161102

Hempel, M., Fischer, T., Schaffner-Bielich, J., \& Liebendörfer, M. 2012, ApJ, 748, 70

Hempel, M., \& Schaffner-Bielich, J. 2010, Nucl. Phys. A, 837, 210

Hempel, M., Schaffner-Bielich, J., Typel, S., \& Röpke, G. 2011, Phys. Rev. C, 84, 055804

Hix, W. R., Messer, O. E., Mezzacappa, A., et al. 2003, Physical Review Letters, 91, 201102

Horowitz, C. 2002, Phys.Rev., D65, 043001

Khan, E., \& Margueron, J. 2012, arXiv:1204.0399

Lalazissis, G. A., König, J., \& Ring, P. 1997, Phys. Rev. C, 55, 540

Langanke, K., Martinez-Pinedo, G., Sampaio, J., et al. 2003, Phys. Rev. Lett., 90, 241102

Lattimer, J. M., \& Lim, Y. 2012, arXiv:1203.4286

Lattimer, J. M., \& Swesty, F. 1991, Nucl. Phys. A, 535, 331

LeBlanc, J., \& Wilson, J. 1970, ApJ, 161, 541

Liebendoerfer, M., Messer, O., Mezzacappa, A., et al. 2004, ApJS, 150, 263

Marek, A., Janka, H.-T., \& Müller, E. 2009, A\&A, 496, 475

Mezzacappa, A., \& Bruenn, S. 1993a, Astrophys.J., 410, 740
-. 1993b, Astrophys.J., 405, 637

Mezzacappa, A., \& Messer, O. E. B. 1999, Journal of

Computational and Applied Mathematics, 109, 281

Mohr, P. J., Taylor, B. N., \& Newell, D. B. 2008, Rev. Mod. Phys., 80, 633

Möller, P., Nix, J. R., Myers, W. D., \& Swiatecki, W. J. 1995,

Atomic Data and Nuclear Data Tables, 59, 185

Nakazato, K., Sumiyoshi, K., \& Yamada, S. 2008, Phys. Rev. D, 77,103006

Nakazato, K., Sumiyoshi, K., \& Yamada, S. 2010, ApJ, 721, 1284

O'Connor, E., \& Ott, C. D. 2011, ApJ, 730, 70

Piekarewicz, J. 2010, J. Phys. G, 37, 064038

Qin, L., Hagel, K., Wada, R., et al. 2012, Phys. Rev. Lett., 108, 172701

Reddy, S., Prakash, M., \& Lattimer, J. M. 1998, Phys. Rev. D, 58, 013009

Reddy, S., Prakash, M., Lattimer, J. M., \& Pons, J. A. 1999, Phys. Rev. C, 59, 2888

Roberts, L. F., Shen, G., Cirigliano, V., et al. 2012, Physical

Review Letters, 108, 061103

Sagert, I., Fischer, T., Hempel, M., et al. 2009, Phys. Rev. Lett., 102,081101

Shen, G., Horowitz, C. J., \& O'Connor, E. 2011a, Phys. Rev. C, 83,065808

Shen, G., Horowitz, C. J., \& Teige, S. 2011b, Phys. Rev. C, 83 , 035802

Shen, H., Toki, H., Oyamatsu, K., \& Sumiyoshi, K. 1998, Nucl. Phys. A, 637, 435

Steiner, A. W.. \& Gandolfi, S. 2012, Phys. Rev. Lett., 108, 081102

Steiner, A. W., Lattimer, J. M., \& Brown, E. F. 2010, ApJ, 722, 33

. 2012, arXiv:1205.6871

Steiner, A. W., Prakash, M., Lattimer, J. M., \& Ellis, P. J. 2005, Phys. Rep., 411, 325

Sugahara, Y., \& Toki, H. 1994, Nucl. Phys. A, 579, 557

Suleimanov, V., Poutanen, J., Revnivtsev, M., \& Werner, K. 2011, ApJ, 742, 122

Sumiyoshi, K., Suzuki, H., \& Toki, H. 1995, å, 303, 475

Sumiyoshi, K., Yamada, S., \& Suzuki, H. 2007, ApJ, 667, 382

Sumiyoshi, K., Yamada, S., Suzuki, H., \& Chiba, S. 2006, Phys. Rev. Lett., 97, 091101 
Tews, I., Krueger, T., Hebeler, K., \& Schwenk, A. 2012, arXiv: 1206.0025

Timmes, F. X., \& Arnett, D. 1999, ApJS, 125, 277

Todd-Rutel, B. G., \& Piekarewicz, J. 2005, Phys. Rev. Lett., 95, 122501

Toki, H., Hirata, D., Sugahara, Y., Sumiyoshi, K., \& Tanihata, I. 1995, Nucl. Phys. A, 588, 357
Tsang, M. B., Zhang, Y., Danielewicz, P., et al. 2009,

Phys. Rev. Lett., 102, 122701

Tsang, M. B., Stone, J. R., Camera, F., et al. 2012,

arXiv: 1204.0466

Woosley, S., Heger, A., \& Weaver, T. 2002, Rev. Mod. Phys., 74, 1015

Woosley, S., \& Weaver, T. 1995, ApJS, 101, 181 